\title{
Tromboembolismo pulmonar
}

\section{Pulmonary thromboembolism}

\author{
Gustavo J. Volpe ${ }^{1,2}$, Luis F. Joaquim ${ }^{1,3}$, Larissa B. A. Dias ${ }^{1}$, Marcelo B. de Menezes ${ }^{4}$, Julio C. Moriguti ${ }^{5}$
}

\begin{abstract}
RESUMO
O tromboembolismo pulmonar (TEP) tem quadro clínico bastante variável, que vai desde quadros completamente assintomáticos, nos quais o diagnóstico é feito incidentalmente, até situações em que êmbolos maciços levam o paciente rapidamente à morte. Seu diagnóstico é difícil e depende da análise da probabilidade pré-teste para otimização da acurácia dos métodos diagnósticos complementares. A pedra angular do tratamento é anticoagulação, porém a trombólise química deve ser considerada em casos de instabilidade hemodinâmica.
\end{abstract}

Palavras-chave: Tromboembolismo Pulmonar. Manifestações Clínicas. Trombólise Terapêutica. Anticoagulantes.

\section{Introdução}

O tromboembolismo pulmonar (TEP) é matéria de interesse de todas as especialidades médicas. De quadro clínico variável, vai desde quadros completamente assintomáticos, nos quais o diagnóstico é feito incidentalmente, até situações em que êmbolos maciços levam o paciente rapidamente à morte. No Brasil não há dados concretos sobre a sua real incidência, mas nos EUA e na Europa estima-se que 200.000 a 300.000 pessoas morram todos os anos com TEP, não sendo raras as situações onde o diagnóstico é feito apenas na necropsia. ${ }^{1 / 5}$
Sua fisiopatologia está intrinsecamente ligada à da trombose venosa profunda (TVP), pois aproximadamente $79 \%$ dos pacientes com TEP têm evidência de TVP nos membros inferiores 1 e 50\% dos pacientes com TVP apresentam embolização pulmonar. $^{6}$ Sendo assim, os fenômenos tromboembólicos (TVP+TEP) compartilham os mesmos fatores de risco. O último Guideline da European Society of Cardiology (ESC) de 2008 traz uma interessante divisão destes fatores, separando-os de acordo com o grau de predisposição de risco e com o fato de estarem relacionados à situação clínica ou ao paciente propriamente dito (Tabela 1).
1 Médico Assistente da Área de Clínica Médica do Hospital Estadual de Ribeirão Preto. 2Pós-graduando da Área de Clínica Médica da FMRP-USP.

3 Mestre em Fisiologia Cardiovascular pela Área de Fisiologia da FMRP-USP.

4 Médico Assistente do Serviço de Pneumologia do Hospital das Clínicas da FMRP-USP.

5 Diretor de Atividades Clínicas do Hospital Estadual de Ribeirão Preto e Professor Livre-Docente da Divisão de Clínica Médica Geral e Geriatria da FMRP-USP.
Correspondência: Gustavo Jardim Volpe Hospital Estadual de Ribeirão Preto Avenida Independência, 4750. 14026-160- Ribeirão Preto - SP Telefone: 16-3602-7100. 


\section{Tabela 1}

Fatores de Risco para Tromboembolismo Venoso. ${ }^{8}$

\begin{tabular}{|c|c|c|}
\hline Fator & Relacionado ao paciente & Relacionado \\
\hline \multicolumn{3}{|c|}{ Alta predisposição (odds ratio > 10) } \\
\hline Fratura da perna ou quadril & & $\mathrm{X}$ \\
\hline Prótese de joelho ou quadril & & $\mathrm{X}$ \\
\hline Cirurgia geral maior & & $\mathrm{X}$ \\
\hline Traumas maiores & & $\mathrm{X}$ \\
\hline Lesão medula espinhal & & X \\
\hline
\end{tabular}

\section{Moderada Predisposição (odds ratio 2-9)}

Cirurgia de joelho por artroscopia

Cateter Venoso central

Quimioterapia

Insuficiência respiratória ou cardíaca crônica

$\mathrm{X}$

Terapia de reposição hormonal $\quad \mathrm{X}$

Câncer

\section{$\mathrm{X}$}

Contraceptivos orais

$\mathrm{X}$

AVC com paralisia

$\mathrm{X}$

Puerpério

TEV prévio

$\mathrm{X}$

Trombofilia

$\mathrm{X}$

\section{Baixa predisposição (odds ratio $<2$ )}

Repouso no leito $>3$ dias

Imobilidade por tempo prolongado (ex. longas viagens)

$\mathrm{X}$

Idade avançada

$\mathrm{X}$

Cirurgia vídeo-laparoscópica

$\mathrm{X}$

Obesidade

$$
\mathrm{X}
$$

Gravidez (pré-parto)

$\mathrm{X}$

Varicosidade de veias

$\mathrm{X}$

AVC - Acidente Vascular Cerebral / TEV - Tromboembolismo venoso

Observando tais dados, vemos que a clássica Tríade de Risco de Virchow (estase sanguínea, lesão vascular e hipercoagulabilidade) reflete a importância da genética e dos fatores ligados ao ambiente, na gênese do tromboembolismo venoso (TEV). ${ }^{7}$ Os fatores relacionados ao ambiente em sua maioria estão ligados aos procedimentos cirúrgicos, ou seja, são temporários e sujeitos a profilaxia. Nestes casos, quando as medidas adequadas são tomadas, o risco de eventos tromboembólicos diminui consideravelmente. ${ }^{8} \mathrm{~A}$ Tabela 2 mostra os principais fatores hereditários ligados à trombofilia. 


\section{Tabela 2}

Fatores hereditários ligados à trombofilia

Deficiência de Antitrombina

Deficiência de Proteína C

Deficiência de Proteína S

Fator V Leiden

Resistência à proteína $\mathrm{C}$ ativada sem fator V Leiden

Mutação no Gene da Protrombina

Disfibrinogenemia

Deficiência de Plasminogênio

O conhecimento de quais fatores predisponentes estão presentes é de grande valia, no entanto em até $30 \%$ dos casos de TEP nenhum deles é encontrado. ${ }^{8}$

\section{Fisiopatologia}

As repercussões do TEP são primariamente hemodinâmicas e surgem quando mais que 30 a $50 \%$ do leito arterial pulmonar está ocluído. ${ }^{9}$ Êmbolos grandes ou múltiplos podem aumentar abruptamente a pressão na artéria pulmonar até níveis não tolerados pelo ventrículo direito (VD), levando rapidamente a morte por dissociação eletro-mecânica. Mesmo que a obstrução não seja maciça, o paciente pode apresentar síncope e/ou hipotensão devido ao baixo débito, secundário à falência do VD ou à insuficiência diastólica do ventrículo esquerdo causada pelo movimento discinético do septo interventricular, consequente à sobrecarga pressórica do VD (Figura 1).

Nos pacientes que sobrevivem a estes eventos iniciais, mecanismos de compensação são ativados. Há estimulação simpática, que leva entre outras coisas ao aumento do inotropismo e cronotropismo cardíacos, que associados ao mecanismo de Frank-Starling e à vasoconstrição periférica podem melhorar o fluxo na circulação pulmonar e consequentemente melhorar a pressão arterial sistêmica. Nova deterioração hemodinâmica pode ocorrer precocemente como resultado de nova embolização ou insuficiência do VD, principalmente quando o quadro não é diagnosticado ou adequadamente tratado. ${ }^{8}$

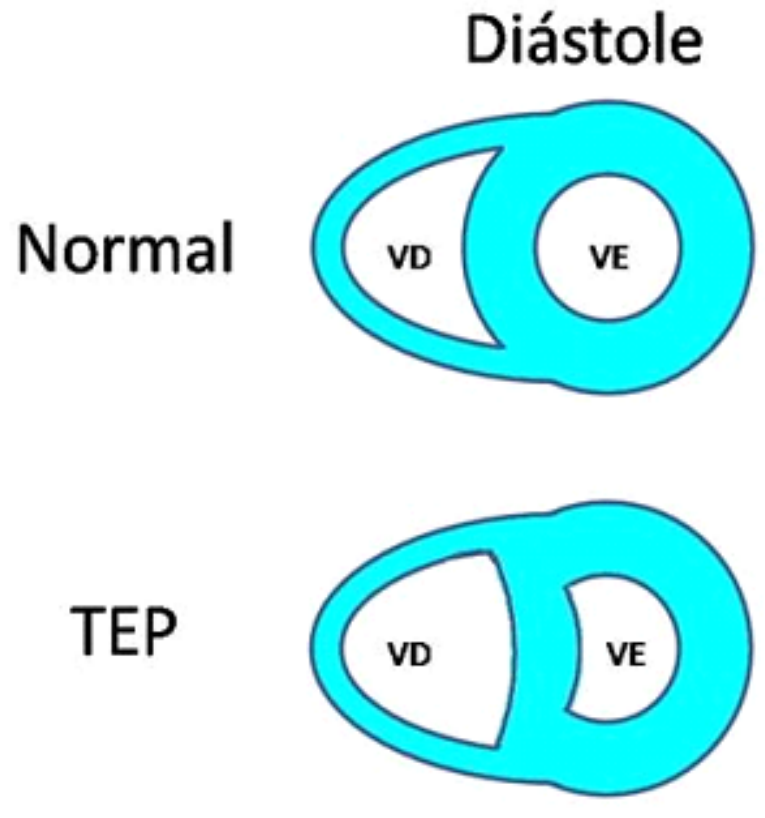

\section{Sístole}
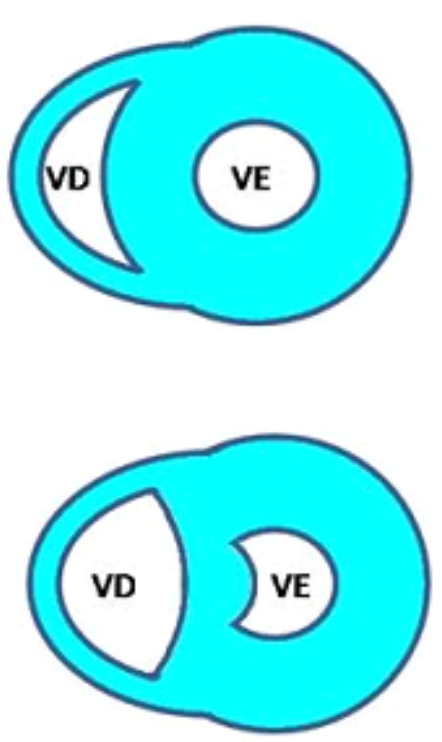

Figura 1: Ilustração de como a sobrecarga aguda do Ventrículo Direito (VD) pode mudar a geometria do Ventrículo Esquerdo (VE). ${ }^{10}$ Acima, em condições fisiológicas durante a sístole há uma redução de volume em ambos os ventrículos, com a contração do VD não alterando a conformação geométrica do VE. Abaixo, quando há sobrecarga do VD em decorrência de TEP, o septo interventricular é rebatido para a esquerda, levando a diminuição do enchimento do VE e perda no recrutamento do mecanismo de Frank-Starling devido a mudança na geometria da câmara. 
A insuficiência respiratória acontece essencialmente em consequência do quadro circulatório. $\mathrm{O}$ baixo débito do VD e a presença de áreas mal perfundidas e normalmente ventiladas (efeito espaço morto) são os principais mecanismos que levam a hipoxemia (Figura 2). Hemorragia e edema alveolar, shunt intracardíaco, redução na produção do surfactante pulmonar, atelectasia e broncoconstrição podem estar associados e também comprometem a oxigenação. O infarto pulmonar é menos frequente, dada a irrigação dupla (artéria pulmonar e artérias brônquicas) do tecido pulmonar. Acontece geralmente quando há pequenos êmbolos distais ou baixo fluxo nas artérias brônquicas (ex. aterosclerose grave) ${ }^{8}$

\section{Apresentação Clínica}

Como já afirmado acima, o espectro da apresentação clínica é bastante amplo. Da mesma forma, os sinais e sintomas são inespecíficos. No entanto eles são importantes não só para sugerir o diagnóstico como também para ajudar na estratificação de risco.

Em 90\% dos casos a hipótese de TEP é sugerida pela presença de dispneia, síncope, dor torácica e taquipneia, sozinhos ou em associação. ${ }^{11}$
A dispneia e a dor torácica podem ser súbitas ou ter evolução ao longo de dias ou semanas. ${ }^{7}$

Síncope é rara, no entanto sua presença está relacionada a quadros mais graves que podem se apresentar também com hipotensão e/ou parada cardíaca. ${ }^{8}$

Dor pleurítica e hemoptise estão relacionadas com o infarto pulmonar. Dor precordial anginosa pode estar presente e pode indicar isquemia do ventrículo direito.

Tosse, palpitações e tontura também podem estar presentes, mas são muito inespecíficos e eventualmente são secundários às doenças associadas.

Taquipneia e taquicardia são os achados de exame físico mais prevalentes. Já os sinais de hipertensão pulmonar (veias do pescoço túrgidas, P2 hiperfonética e impulso do VD palpável) são mais raros, porém mais específicos. Vale lembrar que dada a forte associação entre as condições, sinais e sintomas de TVP (dor, edema, empastamento muscular, etc) também devem ser pesquisados. ${ }^{7}$ Um resumo dos principais sinais e sintomas e suas respectivas prevalências estão na Tabela 3.

Os achados clínicos geralmente estão ligados à carga trombótica do TEP, no entanto não são raras as situações em que grandes êmbolos estão associados
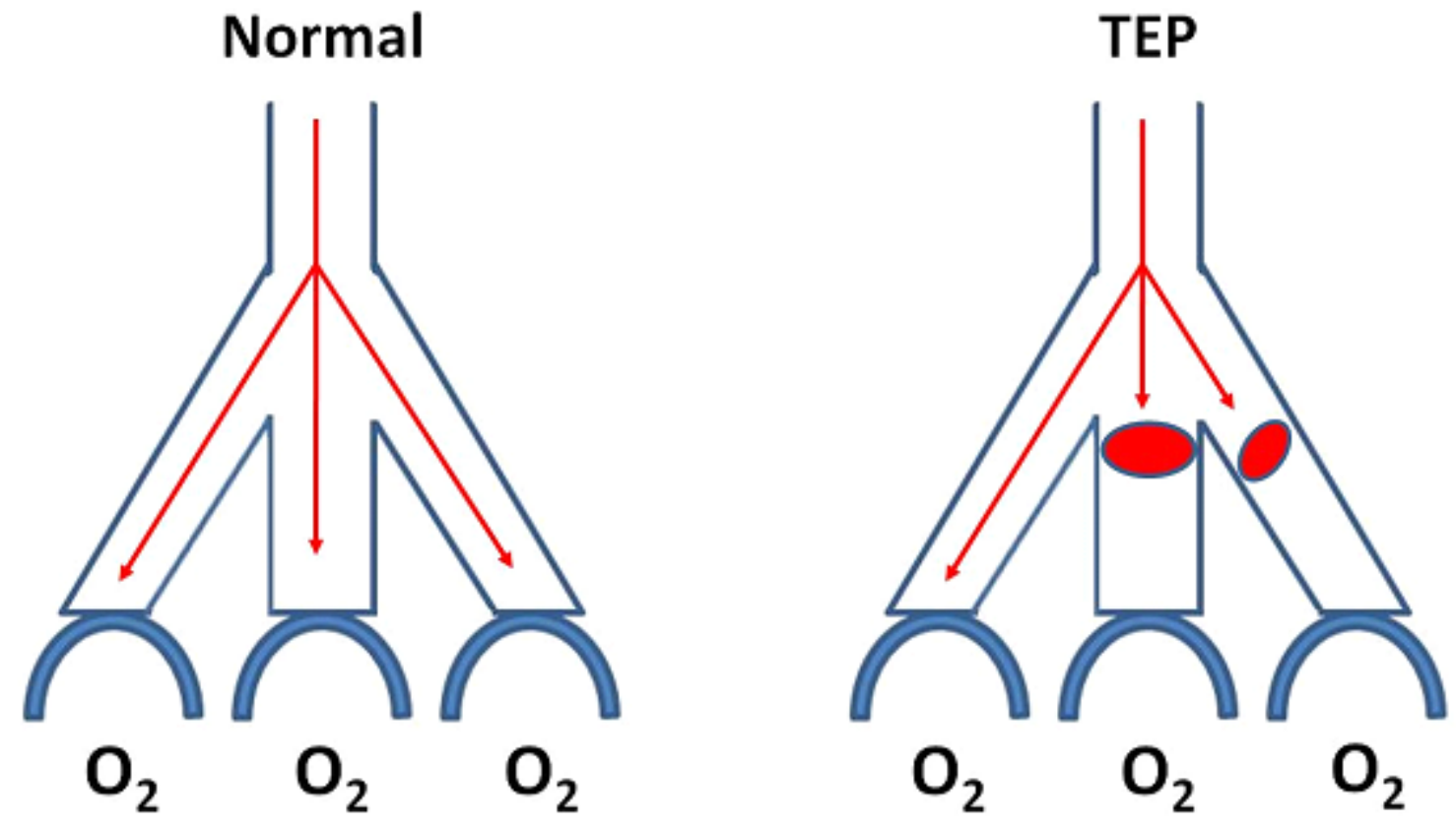

Figura 2: Efeito Espaço Morto

À esquerda observamos o funcionamento esquemático do lóbulo pulmonar. O sangue (setas) chega até o alvéolo (semicírculos), onde é oxigenado. Em situações em que há TEP ocorre o chamado efeito espaço morto (direita), com alvéolos ventilados e não perfundidos devido à presença de trombos na luz do vaso (elipses). Este efeito prejudica a oxigenação sanguínea e pode levar à hipoxemia grave. 


\section{Tabela 3}

Prevalência dos sinais e sintomas em pacientes com suspeita de TEP de acordo com o diagnóstico final, em $\%{ }^{8}$

\begin{tabular}{lcc}
\hline Sintomas & $\begin{array}{c}\text { TEP confirmado } \\
(\mathrm{n}=219)\end{array}$ & $\begin{array}{c}\text { TEP excluído } \\
(\mathrm{n}=546)\end{array}$ \\
\hline Dispneia & 80 & 59 \\
Dor torácica (pleurítica) & 52 & 43 \\
Dor torácica (retroesternal) & 12 & 8 \\
Tosse & 20 & 25 \\
Hemoptise & 11 & 7 \\
Síncope & 19 & 11 \\
Sinais & & \\
Taquipneia $(\geq 20$ ipm $)$ & 70 & 68 \\
Taquicardia $(>100 \mathrm{bpm})$ & 26 & 23 \\
Sinais de TVP & 15 & 10 \\
Febre & 7 & 17 \\
Cianose & 11 & 9 \\
\hline
\end{tabular}

somente a dispneia e achados inespecíficos, enquanto pequenos trombos periféricos podem ter rica apresentação clínica e bastante comprometimento do estado geral (comum em pacientes com baixa reserva cardiovascular). ${ }^{7}$ A possibilidade de êmbolos maciços deve ser suspeitada em casos que têm início súbito, com síncope ou pré-síncope, hipotensão, hipoxemia grave ou parada cardíaca. ${ }^{5}$

\section{Diagnóstico}

Dado o quadro clínico pouco específico, são necessários exames subsidiários para a confirmação no diagnóstico de TEP.

\section{Exames subsidiários}

A radiografia de tórax é normal na maioria dos casos e os achados mais frequentes (derrame pleural, atelectasia e elevação da cúpula diafragmática) também são pouco específicos. Mais do que sugerir TEP, a principal função da radiografia de tórax é excluir outros diagnósticos possíveis. ${ }^{12}$ Hipoxemia é outro achado frequente, presente em até $80 \%$ dos casos. O eletrocardiograma (ECG) pode ser útil quando demonstra sinais de sobrecarga ventricular direita (in- versão de onda T de V1-V4, padrão "strain" em precordiais anteriores, padrão QR em V1, o clássico padrão S1Q3T3 e bloqueio do ramo direito), principalmente se forem agudos. ${ }^{8}$ Apesar de inespecífico, o achado mais comum do ECG é a taquicardia sinusal.

Há exames mais específicos para diagnóstico de TEP, no entanto o uso destas ferramentas deve ser feito com base numa avaliação da probabilidade préteste, pois o valor preditivo positivo dos exames diagnósticos de que dispomos está intrinsecamente ligado a esta avaliação. Dessa forma, todo paciente com suspeita clínica de TEP deve ser estratificado quanto à probabilidade clínica (ou pré-teste) com base em escores de risco validados (a Tabela 4 apresenta os dois principais). Tanto o Escore de Wells quanto o Escore de Genebra revisado têm poder discriminativo e alocam os pacientes entre os de alta, média e baixa probabilidade clínica. Esta estratificação será importante não só na escolha das ferramentas diagnósticas como também na indicação ou não do tratamento. ${ }^{8}$

\section{Biomarcadores}

Os dímeros D são alguns dos produtos da degradação da fibrina e estão presentes no plasma de indivíduos onde haja ativação dos sistemas de coagulação e fibrinólise. Uma dosagem negativa torna o diagnóstico de TEV bastante improvável. Por outro lado, como a produção desses dímeros está ligada à degradação da fibrina e esta é produzida em vários outros processos (inflamação, câncer, necrose, infecção, etc), a especificidade de uma dosagem elevada de dímeros D para TEP é baixa. Ou seja, a dosagem de dímeros $\mathrm{D}$ tem alto valor preditivo negativo e baixo valor preditivo positivo. Em pacientes com probabilidade baixa ou intermediária ela é importante para exclusão do diagnóstico, no entanto, não confirma em hipótese alguma o diagnóstico de TEP. ${ }^{5,8}$

As troponinas T e I estão elevadas em grandes embolizações, situações em que geralmente há dano ao miocárdio do ventrículo direito pela sobrecarga pressórica súbita. Uma dosagem elevada denota maior gravidade no paciente com diagnóstico de TEP estabelecido, porém não tem papel como ferramenta diagnóstica. O mesmo acontece com o peptídeo natriurético cerebral (BNP), liberado quando há distensão de câmaras cardíacas. Apesar de refletir maior gravidade nos pacientes com TEP, doenças outras como insuficiência cardíaca congestiva ou hipertensão pulmonar primária podem também levar a dosagens elevadas de BNP. ${ }^{7}$ 


\section{Tabela 4}

Escores de avaliação de probabilidade pré-teste de TEP $^{8}$

\section{Escore de Genebra revisado}

\section{Fatores predisponentes}

Idade $>65$ anos

TVP ou TEP prévio

Cirurgia ou fratura no último mês

Neoplasia maligna ativa

+1
+3
+2
+2

$+2$

\section{Sintomas}

Dor unilateral em MI

Hemoptise

Sinais clínicos

FC 75-94 bpm

FC $>95 \mathrm{bpm}$

Edema unilateral em MI associado à dor à palpação venosa

$\begin{array}{ll}+3 & \text { Hemoptise } \\ +2 & \end{array}$

\section{Escore de Wells}

\section{Fatores predisponentes}

$\begin{array}{lr}\text { TVP ou TEP prévio } & +1,5 \\ \text { Cirurgia ou imobilização recente } & +1,5 \\ \text { Câncer } & +1\end{array}$

\section{Sinais clínicos}

$\begin{array}{llr}+3 & \text { FC }>100 \mathrm{bpm} & +1,5 \\ +5 & \text { Sinais clínicos de TVP } & +3 \\ & \text { Outro diagnóstico é menos provável que TEP } & +3\end{array}$

$\begin{array}{lclc}\text { Probabilidade clínica } & \text { Total } & \text { Probabilidade clínica } & \text { Total } \\ \text { Baixa } & 0-3 & \text { Baixa } & 0-1 \\ \text { Intermediária } & 4-10 & \text { Intermediária } & 2-6 \\ \text { Alta } & \geq 11 & \text { Alta } & \geq 7\end{array}$

TVP - Trombose venosa profunda, TEP - tromboembolismo pulmonar, MI - membro inferior, FC - freqüência cardíaca

\section{Exames de Imagem}

Como em aproximadamente $90 \%$ dos casos os tromboembolismos pulmonares são originados nos membros inferiores (MMII), a ultrassonografia dos mesmos (USMI) pode ser utilizada como adjuvante no diagnóstico de TEP. Em mãos experientes, tem sensibilidade de $90 \%$ e especificidade de $95 \%$ para TVP proximal de membros inferiores. Este achado em paciente com suspeita de TEP é suficiente para o início de anticoagulação plena. ${ }^{8}$

Atualmente, a venografia por tomografia computadorizada (TC) tem sido proposta para o diagnóstico de TVP em pacientes com suspeita de TEP. É re- alizada junto com a angiografia por CT (angio CT) do tórax com uma mesma injeção de contraste e tem sensibilidade e especificidade equivalentes às do USMI, porém acrescenta grande quantidade de radiação ao exame. Ambos os exames podem ser utilizados para reduzir o número de falsos negativos quando a angio CT é feita em aparelhos com um detector apenas. Porém, quando aparelhos com múltiplos detectores (MDCT) são empregados, a associação de métodos acrescenta pouco à sensibilidade. Nestes casos, dado à radiação adicional e ao pobre benefício, a venografia por CT não deve ser utilizada. ${ }^{8}$

A cintilografia ventilação-perfusão (cintilo V/Q) é um dos métodos diagnósticos validados pela litera- 
tura para confirmação de TEP. É realizado pela comparação entre imagens obtidas após infusão endovenosa de albumina marcada com tecnécio (perfusão) e outras realizadas após inalação de gases radiotraçadores, por exemplo o Xenônio 133 (ventilação). Áreas ventiladas e não perfundidas são suspeitas de TEP. O resultado do exame é classificado pelos critérios do PIOPED Trial ${ }^{13}$ em quatro categorias: normal, baixa probabilidade, intermediária (ou não diagnóstica) e alta probabilidade de TEP.

Enquanto uma cintilografia V/Q considerada normal é suficiente para a exclusão do diagnóstico de TEP em qualquer situação, o achado de alta probabilidade só firma o diagnóstico quando associado à alta probabilidade clínica. Em pacientes com probabilidade clínica baixa e cintilografia V/Q de alta probabilidade, outros testes podem ser necessários para a confirmação. Em qualquer outro cenário de associação de probabilidades ela não é diagnóstica e demanda outros exames. ${ }^{7,8}$

A angio CT de tórax é outro teste confirmatório de TEP. Tem numerosas vantagens sobre a cintilogafia $\mathrm{V} / \mathrm{Q}$, entre elas a velocidade, caracterização de estruturas não vasculares, exclusão de outros diagnósticos diferenciais e detecção de trombose venosa quando associada a venografia por CT. Tomógrafos modernos são capazes até mesmo de evidenciar trombos nas artérias pulmonares segmentares. ${ }^{7}$ Suas grandes desvantagens são a exposição à maior quantidade de radiação (Tabela 5) e o custo.

\section{Tabela 5}

Dose de radiação relacionada a exames radiológicos ${ }^{12}$

\begin{tabular}{lc}
\hline Exame & Dose Efetiva (mSv) \\
\hline Raio X Tórax PA+P & 0,07 \\
Cintilografia V/Q & $1,2-2,0$ \\
Angiografia por CT (tórax) & $1,6-8,3$ \\
Venografia por CT & 5,7 \\
Arteriografia com subtração digital & $3,2-30,1$ \\
\hline
\end{tabular}

Existem ainda diferenças quando ao tomógrafo utilizado: os aparelhos mais antigos, com apenas um detector, não são capazes por si sós de excluir o diagnóstico de TEP e demandam exames complementares para tal. Já a angio CT realizada nos aparelhos mais recentes, os chamados multidetectores, é consi- derada o exame de escolha para o diagnóstico de TEP, com sensibilidade de $83 \%$ e especificidade acima de $90 \%{ }^{8}$ Um exame negativo exclui o diagnóstico em paciente com probabilidade pré-teste baixa ou intermediária, assim como um exame positivo (com trombo evidenciado proximal à artéria lobar) é definitivo para TEP. No entanto, nos raros casos em que há exames negativos na presença de probabilidade clínica alta, testes adicionais (cintilo V/Q, US MI ou mesmo arteriografia pulmonar) devem ser considerados para definição do diagnóstico..$^{8,14}$

A arteriografia pulmonar foi por muito tempo o exame de escolha para diagnóstico do TEP. No entanto, perdeu muito espaço na clínica diária com a evolução da angio CT. Também envolve o uso de contraste venoso e expõe o paciente a grande quantidade de radiação, com a desvantagem de ser um exame invasivo que necessita da cateterização das artérias pulmonares. Seu uso está hoje reservado a casos selecionados, nos quais há dúvida diagnóstica ou a realização dos outros exames não é possível.

A ecocardiografia tem papel fundamental na avaliação do paciente hemodinamicamente instável (choque ou hipotensão). Nesta situação, quando não são encontrados sinais de sobrecarga e/ou disfunção do ventrículo direito o TEP pode ser excluído como o causador do quadro. Já quando tais sinais estão presentes e na vigência de instabilidade hemodinâmica, existe a indicação de terapêuticas mais agressivas como trombólise química ou tromboembolectomia cirúrgica. Em pacientes hemodinamicamente estáveis, seu papel tende a ser mais como estratificador de risco. Achados de disfunção ou sobrecarga do VD são critérios de pior prognóstico nesta situação. Achado de trombo na artéria pulmonar por este método é raro. ${ }^{8,7}$

Tendo em mente as informações acima, podemos definir as estratégias para cada situação clínica, que são apresentadas nos algoritmos abaixo (Figuras $3,4,5$ e 7). Seu uso contempla a maioria das situações clínicas, porém algumas ressalvas devem ser feitas:

- Doença pulmonar prévia ou mesmo alterações em radiografia de tórax aumentam muito a quantidade de cintilos V/Q inconclusivas ${ }^{15}$;

- Qualquer modalidade de CT deve ser evitada em pacientes com insuficiência renal devido à nefrotoxicidade dos contrastes utilizados;

- Não há qualquer indicação para dosagem de dímeros D em pacientes com instabilidade hemodinâmica e/ ou alta probabilidade clínica de $\mathrm{TEP}^{5,8}$; 


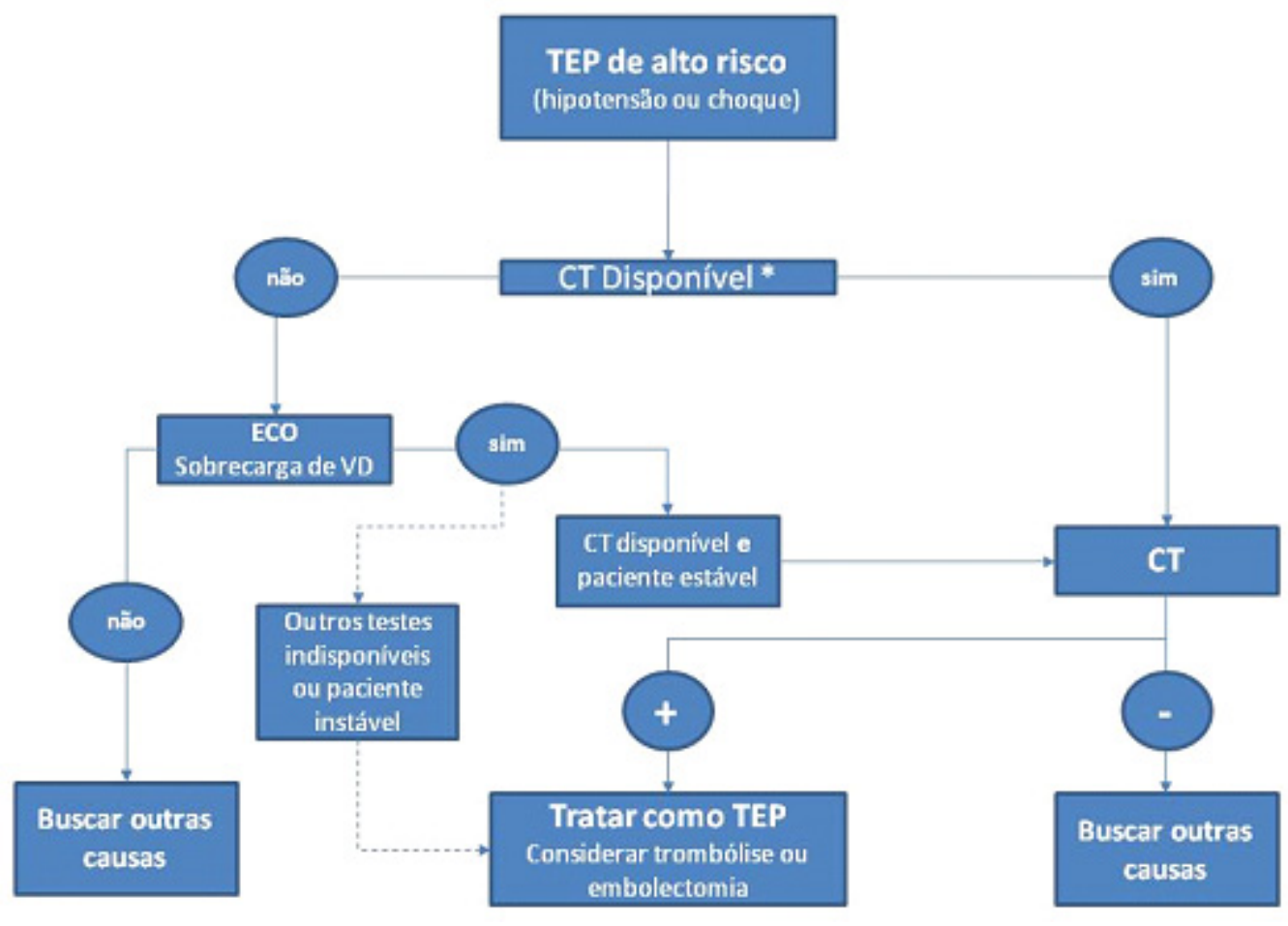

Figura 3: Fluxograma para TEP de alto risco (instabilidade hemodinâmica). ${ }^{8}$

${ }^{*}$ CT disponível também considerando se paciente tem condições clínicas para o exame.

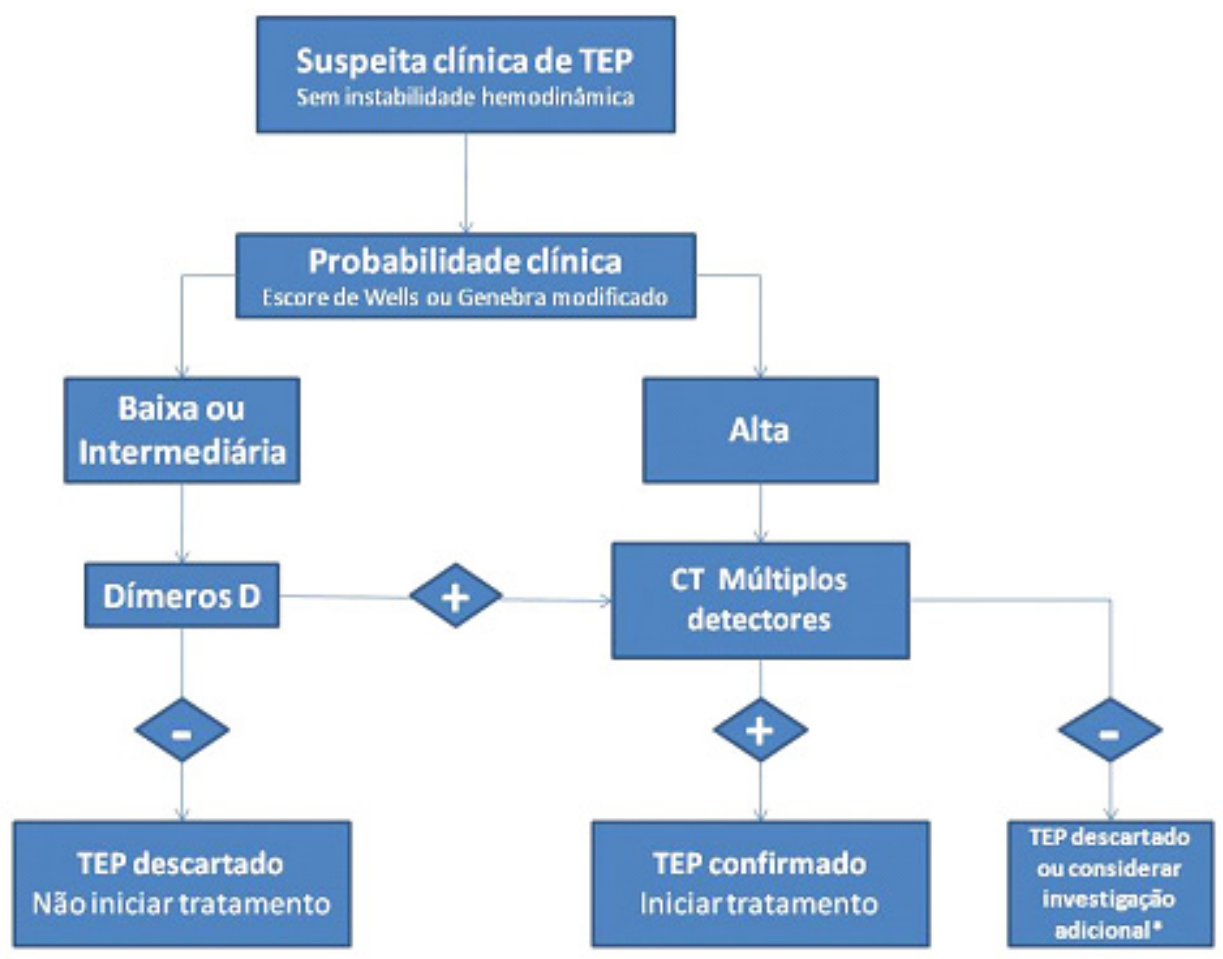

Figura 4: Fluxograma para TEP não alto risco e com uso de Tomografia com múltiplos detectores. ${ }^{8}$

*Nesta situação outros exames como cintilografia V/Q ou Ultrassonografia de Membros inferiores podem ser realizados para confirmar a exclusão do diagnóstico (detalhes no texto). 


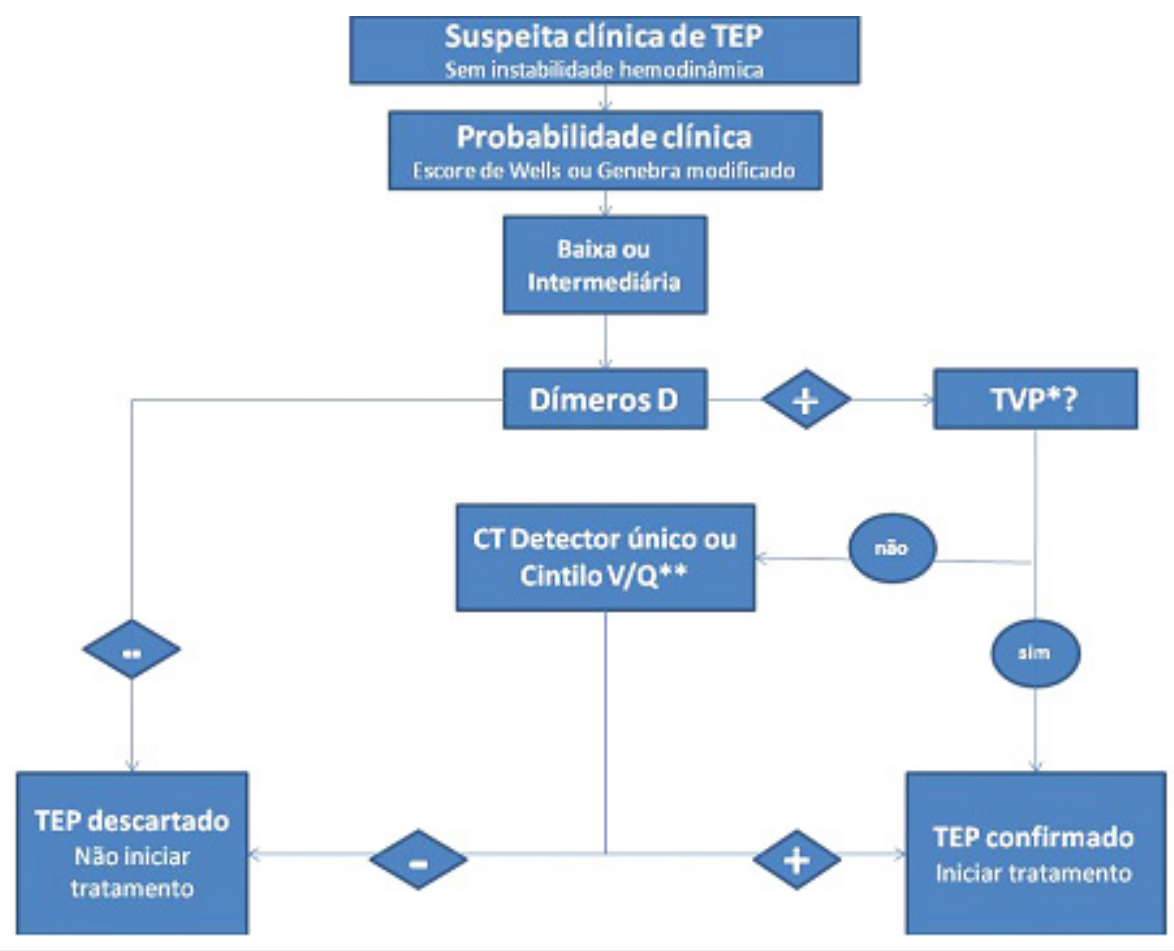

Figura 5: Fluxograma para o diagnóstico de TEP hemodinamicamente estável com uso de tomografia com detector único em pacientes com probabilidade baixa ou intermediária (5).

${ }^{*}$ A pesquisa pode ser tanto realizada com Venografia por CT quando Ultrassonografia de membros inferiores.

${ }^{* *}$ Cintilografia Ventilação/Perfusão quando positiva confirma o diagnóstico tanto com probabilidade baixa ou intermediária, assim como na mesma situação a normal exclui o diagnóstico quando associado à USG de membros inferiores negativo. No entanto, quando inconclusiva, somente excluiu o diagnóstico em situações de baixa probabilidade.

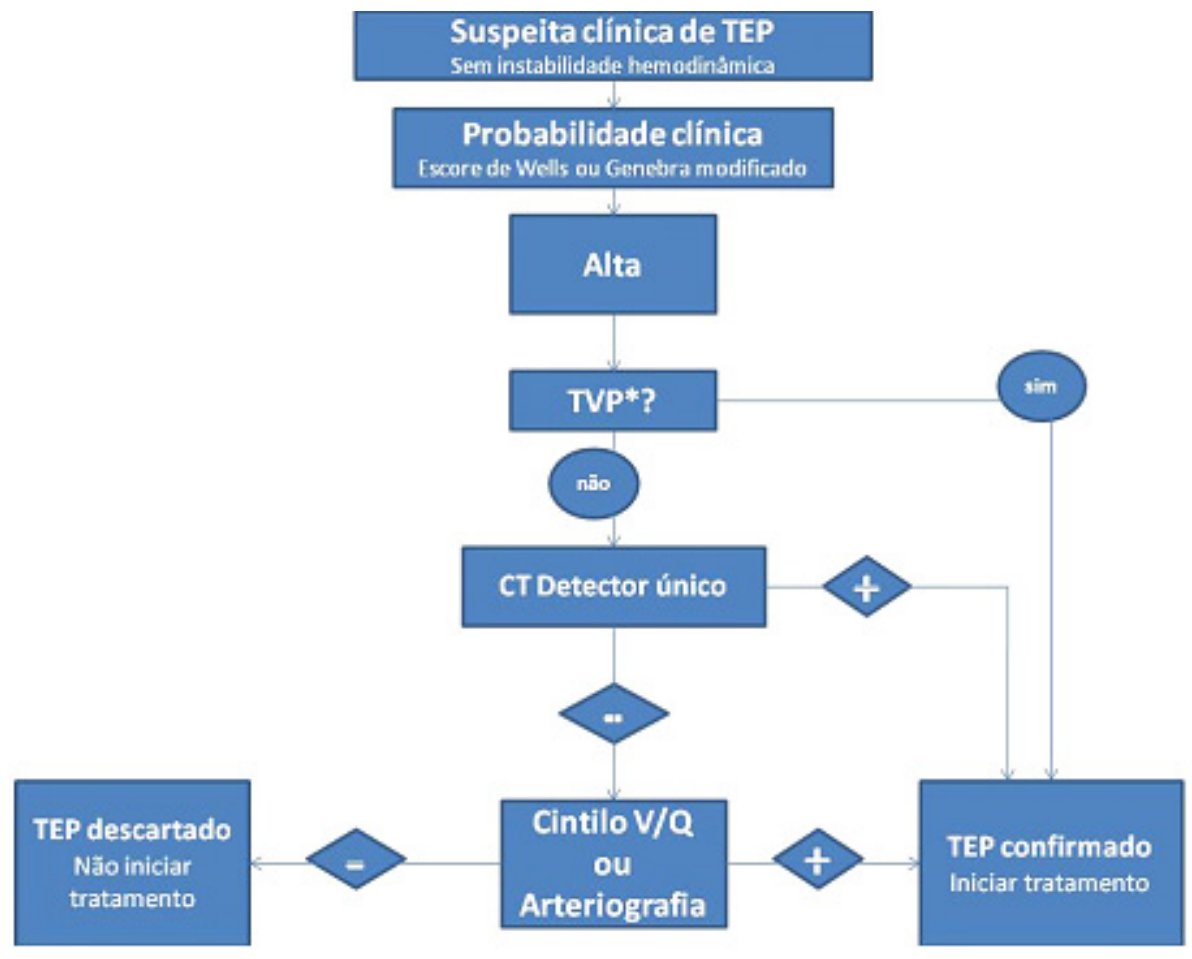

Figura 6: Fluxograma para o diagnóstico de TEP hemodinamicamente estável com uso de tomografia com detector único em pacientes com alta probabilidade (5).

${ }^{*}$ A pesquisa pode ser tanto realizada com Venografia por CT quanto Ultrassonografia de membros inferiores. 


\section{Tratamento}

Pacientes com suspeita de TEP e hemodinamicamente instáveis devem receber suporte hemodinâmico e respiratório adequados. A suplementação de $\mathrm{O}_{2}$ geralmente é necessária para manutenção de saturação $>90 \%$ e/ou $\mathrm{PaO}_{2}>70 \mathrm{mmHg}$, inclusive com uso de ventilação mecânica. Aminas vasoativas como dopamina e dobutamina podem ser utilizadas para a recuperação da pressão e do débito cardíaco, no entanto reposição volêmica deve ser realizada com cautela. Quando realizada de forma agressiva, pode levar a diminuição ainda maior do débito cardíaco devido à dilatação do ventrículo direito (Figura 1). ${ }^{8,10}$

A anticoagulação plena é a pedra angular do tratamento do TEP e deve ser iniciada tão logo seja sugerido o diagnóstico e a probabilidade clínica seja intermediária ou alta, dada a alta mortalidade relacionada a pacientes não tratados adequadamente. ${ }^{17}$

A rápida obtenção de anticoagulação plena depende do uso de medicações parenterais, principalmente a heparina não fracionada e as de baixo peso molecular. $\mathrm{O}$ fondaparinux também pode ser utilizado, porém até o momento não está disponível no Brasil. Pacientes hemodinamicamente instáveis devem receber preferencialmente heparina não fracionada, uma vez que as heparinas de baixo peso molecular não foram validadas até o momento para este uso. ${ }^{8}$ Antagonistas da vitamina $\mathrm{K}$ devem ser iniciados junto com os agentes parenterais para obtenção precoce de anticoagulação oral efetiva, o que possibilita menor tempo de internação. O tempo de protrombina alvo corresponde a INR entre 2,0 e $3,0 .{ }^{8} \mathrm{~A}$ anticoagulação plena segue os protocolos habituais e seu manejo pormenorizado será abordado em outro texto deste mesmo simpósio.

Pacientes com TEP hemodinamicamente instáveis devem receber tratamento com trombolíticos o mais precocemente possível, preferencialmente nas primeiras 48 horas. No entanto, se quadro clínico persistir, pode ser realizado até 7 a 14 dias após o evento agudo. ${ }^{8}$ Os regimes de trombolíticos aprovados para uso no TEP podem ser vistos na Tabela 7. Heparinização plena deve ser feita concomitante ao ativador tissular do plasminogênio recombinante (rTPA), porém não deve ser realizada em associação com estreptoquinase e uroquinase pelo alto risco de sangramento.

As contraindicações relativas e absolutas são a princípio semelhantes às utilizadas no infarto agudo do miocárdio (Tabela 7), porém ressalta-se que mesmo as consideradas absolutas podem ser relativas em pacientes com risco iminente de morte. ${ }^{8}$

\section{Tabela 6}

Trombolíticos recomendados no Tromboembolismo Pulmonar

Estreptoquinase $250.000 \mathrm{UI}$ em 30 minutos seguidos por infusão de $100.000 \mathrm{UI} / \mathrm{h}$ por $12 \mathrm{a} 24$ horas

Protocolo acelerado: 1.500.000 UI em 2 horas

Uroquinase $\quad 4.400 \mathrm{UI} / \mathrm{Kg}$ em 10 minutos, seguido por $4.400 \mathrm{UI} / \mathrm{Kg} / \mathrm{h}$ por $12 \mathrm{a} 24$ horas

Protocolo acelerado: 3.000 .000 UI em 2 horas

rTPA $\quad 100 \mathrm{mg}$ em 2 horas ou $0,6 \mathrm{mg} / \mathrm{Kg}$ em 15 minutos (máximo $50 \mathrm{mg}$ )

rTPA - Ativador tissular da plasmina recombinante

\section{Tabela 7}

Contra-indicações ao uso de Trombolíticos

\section{Absolutas*}

AVC hemorrágico ou AVC de origem desconhecida pregresso

AVC sabidamente isquêmico nos últimos 6 meses

Neoplasia ou lesões encefálicas

Cirurgia ou Trauma ou TCE maior nas últimas 3 semanas Hemorragia gastrointestinal no último mês

Sangramento ativo

\section{Relativas}

AIT nos últimos 6 meses

Anticoagulação plena

Gravidez ou $1^{\circ}$ mês do puerpério

Punções não compressíveis

Manobras de ressuscitação cardiopulmonar traumáticas

Hipertensão refratária (PA sistólica > 180 mmHg)

Insuficiência hepática

Endocardite infecciosa

Úlcera péptica ativa

AVC - acidente vascular cerebral, TCE - traumatismo crânioencefálico, AIT - acidente isquêmico transitório 
As embolectomias cirúrgica e percutânea também são tratamentos válidos, com sua principal indicação nos pacientes que apresentam contra-indicação ao uso de fibrinolíticos ou mesmo sem resposta à sua infusão. Em pacientes hemodinamicamente estáveis e com sinais de disfunção do VD no ECO, o uso de fibrinolíticos é controverso. Existem somente pequenos trabalhos que sugerem benefício no seu uso e um grande trabalho europeu está em curso para responder a esta questão. Por hora, o Guideline da European Society of Cardiology de 2008 sugere que a trombólise seja realizada nesta situação apenas em casos selecionados. ${ }^{8}$

Após o tratamento inicial, o paciente deve permanecer internado até sua completa estabilização clínica e até que sua anticoagulação com antagonistas da vitamina $\mathrm{K}$ esteja efetiva. Após a alta hospitalar o tratamento deve ser mantido por pelo menos 3 meses naqueles casos sem fator causal aparente ou naqueles em que o fator predisponente era reversível. ${ }^{8} \mathrm{Em}$ casos de recorrência de fenômeno trombótico por motivo inaparente e com fator predisponente irreversível (principalmente genético), recomenda-se anticoagulação plena por tempo indefinido. Paciente com câncer deve receber anticoagulação por 3 a 7 meses no mínimo; no entanto, deve ser considerada sua manutenção por tempo indeterminado nestes casos. ${ }^{8}$

A implantação de filtros de veia cava é terapia alternativa, que só deve ser indicada em casos de contra-indicação absoluta de anticoagulação plena ou naqueles com eventos tromboembólicos de repetição na vigência de anticoagulação efetiva. ${ }^{7}$ Seu uso previne somente a ocorrência de TEP e, no entanto, está relacionada com complicações a longo prazo como síndrome pós trombótica e recorrência de TVP. ${ }^{8}$

\section{Profilaxia}

Dada a grande associação entre os casos de TEV e a imobilização temporária devido à hospitalização, principalmente nos casos de cirurgia, devemos adotar medidas para prevenir tais eventos nestas situações.

Sem profilaxia, a incidência de TVP intra-hospitalar objetivamente confirmada em pacientes clínicos ou de cirurgia geral é aproximadamente 10 a 40\%, enquanto naqueles submetidos a grandes cirurgias ortopédicas gira em torno de 40 a $70 \% .{ }^{19}$ São muitas as evidências da literatura que suportam a profilaxia, demonstrando seu claro benefício. ${ }^{17 / 20}$ Ela pode ser rea- lizada por meio de medidas farmacológicas ou mecânicas. As últimas baseiam-se no uso de dispositivos de compressão ou contra-pulsação de membros inferiores por meio de calças infláveis.

As medidas farmacológicas baseiam-se no uso das heparinas subcutâneas (não fracionadas e de baixo peso molecular - a Tabela 8 mostra as posologias recomendadas). ${ }^{20} \mathrm{~A}$ terapia farmacológica deve ser a preferencial, reservando-se o uso dos dispositivos mecânicos para situações em que haja contra-indicação ao uso de heparina. ${ }^{19}$

\section{Tabela 8}

Doses e mediações na profilaxia do tromboembolismo venoso $^{20}$.

Heparina

(não fracionada) 5000 UI subcutânea, de 8 em 8 horas*

Enoxaparina $\quad 40 \mathrm{mg}$ subcutânea, uma vez ao dia**

Dalteparina $\quad 5000$ UI subcutânea, uma vez ao dia

*Apesar de esta posologia ser a mais recomendada, $5000 \mathrm{UI}$ de 12 em 12 horas também pode ser utilizado, principalmente em pacientes cirúrgicos de risco moderado (ver tabela 9).

** $20 \mathrm{mg}$ uma vez ao dia não é efetivo

Deve-se ter em mente, quando se considera o uso da profilaxia para TEV, que tal procedimento predispõe a complicações (sangramentos, plaquetopenia, etc), aumenta o custo da internação e, portanto, não está indicado em todas as situações. Deve haver seleção dos pacientes levando-se em conta a relação risco x benefício. Na avaliação dessa relação podemos dividir os pacientes entre aqueles hospitalizados por motivos clínicos e aqueles internados por motivos cirúrgicos. Recentemente, o Conselho Federal de Medicina (CFM) em conjunto com a Associação Médica Brasileira (AMB), no Projeto Diretrizes, lançou suas recomendações para pacientes clínicos (Figura 7). ${ }^{17}$

Nos pacientes cirúrgicos a indicação da profilaxia depende do tipo de cirurgia e na presença de fatores de risco (Tabela 9). ${ }^{19}$

As medidas profiláticas devem ser mantidas durante toda a internação e/ou enquanto o paciente mantiver imobilidade (mais que a metade do tempo no leito ou sentado à beira do mesmo, sem contar o período de sono). Mobilização e deambulação precoce devem sempre ser estimuladas quando possível. ${ }^{18,19,20}$ 


\section{Todos os pacientes clínicos devem ser rotineiramente avaliados}

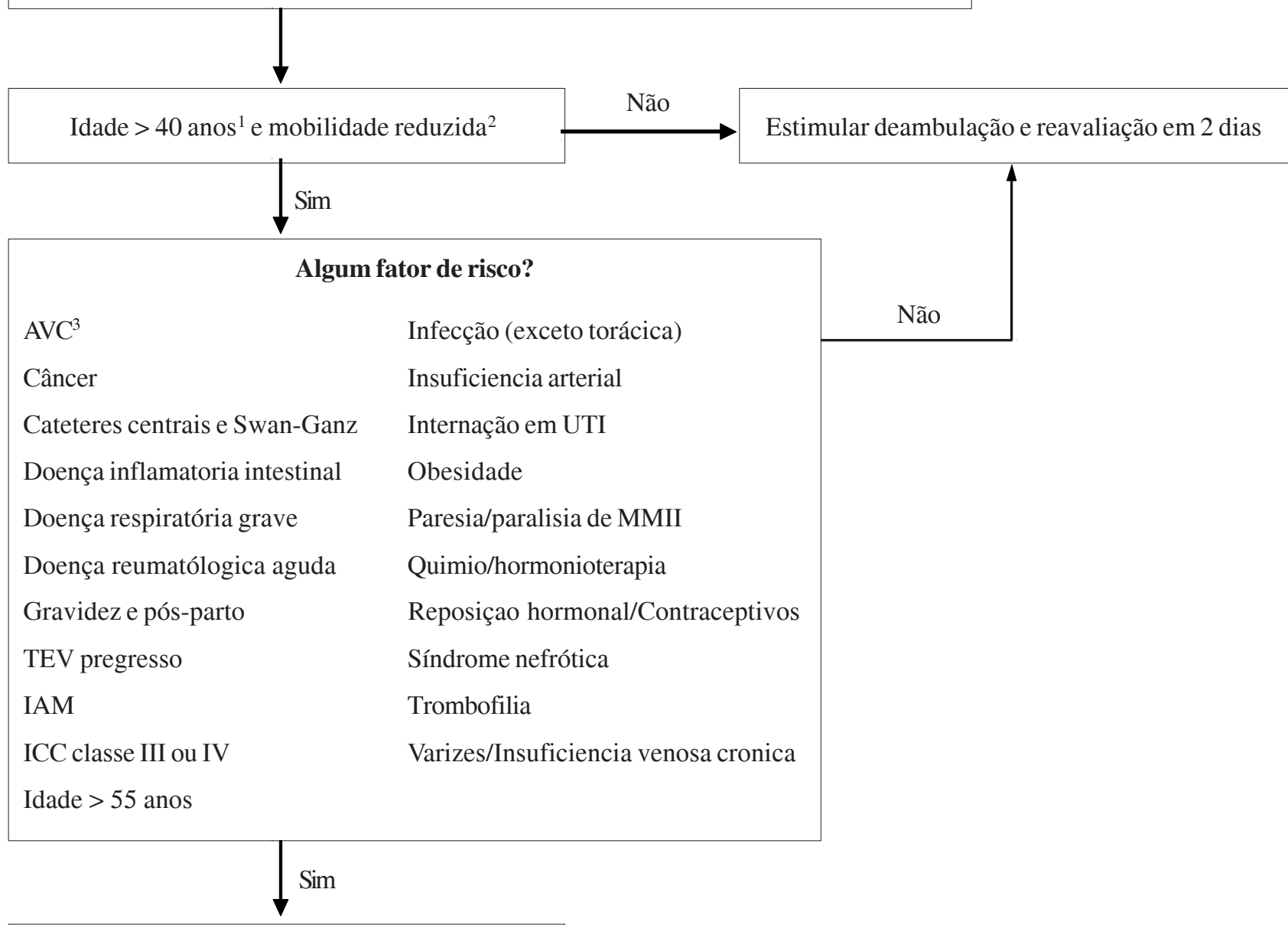

\section{Contra-indicação?}

Sangramento ativo

Úlcera péptica ativa

HAS nao controlada (> $180 \times 110 \mathrm{mmHg}$ )

Coagulopatia (plaquetopenia ou INR $>1,5$ )

Alergia ou plaquetopenia por heparina prévia

Insuficiência renal (clearance $<30 \mathrm{ml} / \mathrm{min})^{4}$

Cirurgia craniana ou ocular $<2$ semanas

Coleta de Líquor $<24$ horas

$\downarrow$ Não

\section{Profilaxia indicada}

1- Pacientes $<40$ anos e com fatores de risco adicionais podem se beneficiar da profilaxia.

2- Pelo menos metade do dia deitado ou sentado à beira do leito (excluído período de sono)

3- AVC isquêmico - excluir hemorragia com CT ou RM.

ACV Hemorrágico - considerar profilaxia após o $10^{\circ}$ dia, com estabilidade clínica e tomográfica

4- Em pacientes de alto risco, a profilaxia pode ser indicada.

Figura 7: Fluxograma para avaliação de profilaxia de TP/TEP em pacientes clínicos (adaptado de referência 17) . 


\title{
Tabela 9
}

Risco de TVP/TEP em pacientes cirúrgicos e recomendações de profilaxia. ${ }^{19}$

\begin{tabular}{|c|c|c|c|c|c|}
\hline \multirow[b]{2}{*}{ Nível de risco } & \multicolumn{2}{|c|}{ TVP, $\%$} & \multicolumn{2}{|c|}{ TEP, $\%$} & \multirow[b]{2}{*}{ Profilaxia } \\
\hline & Distal & Proximal & Clínico & Fatal & \\
\hline BAIXO & 2 & 0,4 & 0,2 & $<0,01$ & \\
\hline $\begin{array}{l}\text { Cirurgia menor em pacientes }<40 \text { anos } \\
\text { sem fatores de risco }\end{array}$ & & & & & $\mathbf{A}$ \\
\hline MODERADO & $10-20$ & $2-4$ & $1-2$ & $0,1-0,4$ & \\
\hline \multicolumn{6}{|l|}{ Cirurgia menor em pacientes com fator de risco } \\
\hline \multicolumn{6}{|l|}{$\begin{array}{l}\text { Cirurgia em pacientes com } 40 \text { a } 60 \text { anos sem } \\
\text { fatores de risco }\end{array}$} \\
\hline ALTO & $20-40$ & $4-8$ & $2-4$ & $0,4-1,0$ & \\
\hline $\begin{array}{l}\text { Cirurgia em pacientes }>60 \text { anos ou com } 40 \\
\text { a } 60 \text { anos e fatores de risco }\end{array}$ & & & & & B \\
\hline MUITO ALTO & $40-80$ & $12-20$ & $4-10$ & $0,2-5$ & \\
\hline \multicolumn{6}{|c|}{ Cirurgia em pacientes com múltiplos fatores de risco } \\
\hline \multicolumn{6}{|l|}{ Artroplastia de joelho ou quadril } \\
\hline \multicolumn{6}{|l|}{ Politrauma maior } \\
\hline Trauma de coluna vertebral & & & & & \\
\hline
\end{tabular}

Esquema A - Sem heparinização profilática, estimular deambulação precoce

Esquema B - Heparinização profilática.

Fatores de Risco: Tromboembolismo venoso prévio, câncer, trombofilia e idade $>60$ anos

\begin{abstract}
Pulmonary embolism (PE) has highly variable clinical presentation, ranging from completely asymptomatic patients, in which the diagnosis is made incidentally, to situations where massive emboli lead the patient quickly to death. Its diagnosis is difficult and depends on analysis of pre-test probability for optimum accuracy of diagnostic procedures. The hallmark of treatment is anticoagulation, but the chemical thrombolysis should be considered in cases of hemodynamic instability.
\end{abstract}

Keywords: Pulmonary Thromboembolism. Signs and Symptoms. Thrombolytic Therapy. Anticoagulants.

\section{Referências Bibliográficas}

1. Sandler DA, Martin JF. Autopsy proven pulmonary embolism in hospital patients:are we detecting enough deep vein thrombosis? J R Soc Med 1989;82:203-5.

2. Silverstein MD, Heit JA, Mohr DN, Petterson TM, O'Fallon WM, Melton LJ III. Trends in the incidence of deep vein thrombosis and pulmonary embolism: a 25-year population-based study. Arch Intern Med 1998;158:585-93.

3. Heit JA, Cohen AT, Anderson FA, VTE Impact Assessment Group. Estimated annual number of incident and recurrent, non-fatal and fatal venous thromboembolism (VTE) events in the U. S. Blood 2005; 107:277a. abstract.

4. Bergqvist D, Lindblad B. A 30-year survey of pulmonary embo- 
lism verified at autopsy: an analysis of 1274 surgical patients. Br J Surg 1985;72:105-8.

5. Konstantinides SV. Acute pulmonary embolism revisited. Heart 2008;94:795-802

6. Moser KM, Fedullo PF, Littlejohn JK, Crawford R. Frequent asymptomatic pulmonary embolism in patients with deep venous thrombosis. JAMA 1994;271:223-25

7. Tapson VF. Acute Pulmonary Embolism. N Engl J Med 2008;358:1037-52.

8. Torbicki A, Perrier A, Konstantinides S, Agnelli G, Galiè N, Pruszczyk $P$ et al. Guidelines on the diagnosis and management of acute pulmonary embolism: the Task Force for the Diagnosis and Management of Acute Pulmonary Embolism of the European Society of Cardiology (ESC). Eur Heart J 2008;29(18):2277-315.

9. McIntyre KM, Sasahara AA. The hemodynamic response to pulmonary embolism in patients without prior cardiopulmonary disease. Am J Cardiol 1971;28:288-94.

10. Greyson CR. Pathophysiology of right ventricular failure. Crit Care Med 2008;37(1 Suppl):S57-75.

11. Miniati M, Prediletto R, Formichi B, Marini C, Di Ricco G, Tonelli $L$ et al. Acuracy of clinical assessment in diagnosis of pulmonary embolism. Am J Resp Crit Care Med 1999;159:87471.

12. Stein PD, Henry JW. Clinical Characteristics of pacientes with acute pulmonary embolism stratified according to their presenting syndromes. Chest 1997;112:974-79.
13. Value of the ventilation/perfusion scan in acute pulmonary embolism. Results of the Prospective Investigation of Pulmonary Embolism Diagnosis (PIOPED). The PIOPED Investigators. JAMA 1990;273:2753-59.

14. Stein PD, Woodard PK, Weg JG, Wakefield TW, Tapson VF, Sostman AH et al. Diagnostic Pathways in Acute Pulmonary Embolism: Recomendations of The PIOPED II Investigators. Am J Med 2007;119:1048-55.

15. Lippmann ML, Fein AM. The diagnosis of acute pulmonary embolism in patients with COPD. Chest 1993;104:983-84.

16. Aujesky D, Jiménez D, Mor MK, Geng M, Fine MJ, Ibrahim SA. Weekend versus weekday admission and mortality after acute pulmonary embolism. Circulation. 2009;119:972-8.

17. Academia Brasileira de Neurologia et al. Tromboembolismo venoso: profilaxia em pacientes clínicos - parte I. Rev Assoc Med Bras 2009;55(2)2:102-5.

18. Thromboembolic Risk Factors (THRIFT) Consensus Group. Risk of and prophylaxis for venous thromboembolism in hospital patients. BMJ 1992;305:577-74.

19. Geerts WH, Pineo GF, Heit JA, Bergqvist D, Lassen MR, Colwell CW et al. Prevention of Thromboembolism. Chest 2004; 127:338S-400S

20. Francis CW. Prophylaxis for thromboembolism in hospitalized Medical Patients. N Engl J Med 2007;357(14):1438-44. 